\title{
A narrative review of indocyanine green near-infrared fluorescence imaging technique: a new application in thoracic surgery
}

\author{
Fudong Wang, Yuan Weng, Jiqun Geng, Jun Zhu \\ Department of Thoracic Surgery, Affiliated Hospital of Jiangnan University, Wuxi, China \\ Contributions: (I) Conception and design: F Wang, Y Weng; (II) Administrative support: Y Weng, J Zhu; (III) Provision of study materials or patients: \\ F Wang, Y Weng, J Geng; (IV) Collection and assembly of data: All authors; (V) Data analysis and interpretation: All authors; (VI) Manuscript \\ writing: All authors; (VII) Final approval of manuscript: All authors. \\ Correspondence to: Jun Zhu. Department of Thoracic Surgery, Affiliated Hospital of Jiangnan University, 200 Huihe Road, Wuxi 214062, China. \\ Email: zhjsurgeon@126.com.
}

\begin{abstract}
In clinical work of thoracic surgery, accurate anatomic location of pulmonary nodules, precise demarcation of segmental and sub-segmental plane, tracing of sentinel lymph nodes (SLNs), protection of thoracic nerve, averting of anastomotic fistula etc. always puzzling the surgeons. Indocyanine green (ICG) is a near-infrared fluorescence (NIRF) contrast agent that is the only reagent approved by the Food and Drug Administration (FDA) for use in human subjects due to its low toxicity and high absorbance in the near-infrared spectrum. The application of ICG NIRF imaging technology in surgery is safe and feasible, it can offer real-time fluorescing information and help surgeons make important decisions during surgery. Many studies demonstrated that ICG NIRF can be used to determine tissue perfusion, lymphatic reflux and other fields. In recent years, it has been reported in the field of thoracic surgery and got success. It can provide more information compared to conventional video-assisted thoracoscopic surgery (VATS). In this review, the research results of this technique in animal models and clinical trials in recent years are reviewed the application of ICG NIRF technique in accurate positioning of pulmonary nodules, precise demarcation of segmental and sub-segmental plane, SLNs tracing, thoracic nerve protection, anastomotic leakage and chylothorax prevention.
\end{abstract}

Keywords: Indocyanine green (ICG); near-infrared; fluorescence imaging; thoracic surgery

Received: 21 December 2019; Accepted: 04 November 2020; Published: 25 November 2021.

doi: $10.21037 /$ ccts-19-84

View this article at: http://dx.doi.org/10.21037/ccts-19-84

\section{Introduction}

With the continuous development of video-assisted thoracoscopic techniques, thoracic surgery has changed from traditional thoracotomy to minimally invasive surgery; there is no doubt that video-assisted thoracoscopic surgery (VATS) has shown its great advantages in clinical practice. However, numerous reports mention many clinical problems, such as small pulmonary nodule localization, sentinel lymph nodes (SLNs) recognition, accurate positioning of the pulmonary segmental and sub-segmental plane, which have been plaguing thoracic surgeons. Compared with conventional thoracotomy, the VATS did not show advantages in the assessment of tissue blood flow perfusion and the control of complications such as anastomotic leakage. Therefore, it is necessary to explore new technologies to conquer these challenges.

Indocyanine green (ICG) is a near-infrared fluorescence (NIRF) contrast agent that is the only reagent approved by the Food and Drug Administration (FDA) for use in human subjects due to its low toxicity and high absorbance in the near-infrared spectrum. It is a water-soluble molecule that binds to plasma proteins immediately after intravenous injection and rapidly distribute through blood circulation. It is efficiently and selectively taken up by the liver cells and passed through the biliary tract. The NIRF imaging 


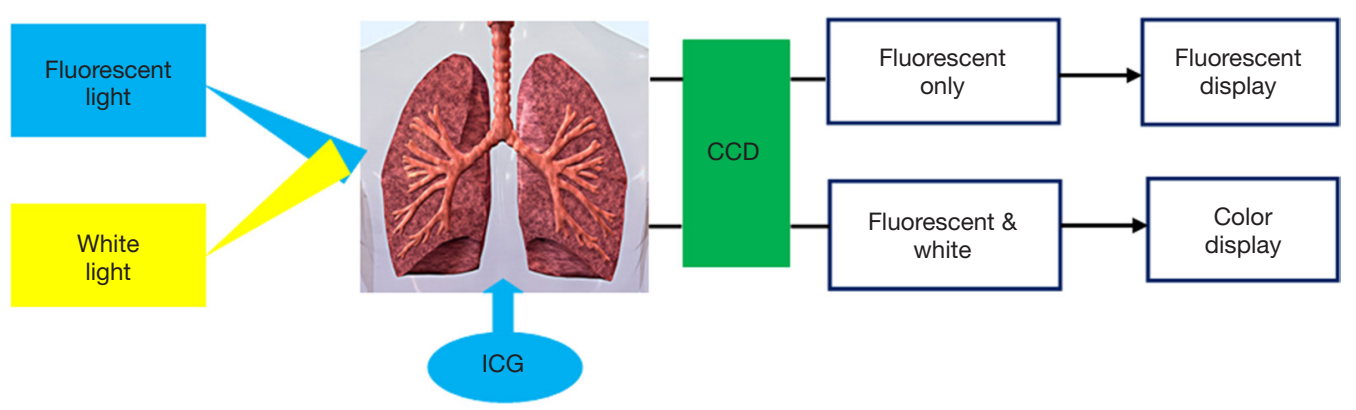

Figure 1 Principle in NIRF thoracoscope. NIRF technology uses a fluorescent contrast (ICG) agent which absorbs near-infrared light, releasing the characteristics of different wavelengths of fluorescence, and then developing on the screen. Fluorescent images are obtained when only the near-infrared light is supplied as illumination, while color images are obtained when the combination of both near-infrared light and white light are supplied as illumination. NIRF, near-infrared fluorescence, ICG, indocyanine green; CCD, charge-coupled device.

system consists of a 785-nm excitation light source, a nearinfrared-sensitive image intensifier, and a customized charge-coupled device (CCD) camera outfitted with filters to collect fluorescence at $830 \mathrm{~nm}$ (Figure 1). Real-time fluorescein imaging technology uses a fluorescent contrast agent which absorbs near-infrared light, releasing the characteristics of different wavelengths of fluorescence, and then developing on the screen. ICG real-time fluorescing imaging technology can provide real-time tissue perfusion image, calibrate specific tissue areas, identify lymph nodes for surgeons. This technique can help surgeons make important decisions during the surgery. The author reviews the application progress of ICG NIRF imaging technology in the above-mentioned fields, and expects to provide new ideas for thoracic surgeons to settle clinical problems. We present the following article in accordance with the Narrative Review reporting checklist (available at https:// ccts.amegroups.com/article/view/10.21037/ccts-19-84/rc).

\section{Methods}

Electronic databases were systematically searched via MEDLINE/PubMed from inception to 2019. The following medical subject headings were searched: "indocyanine green (or ICG)" and "thoracic surgery", "indocyanine green (or ICG)" and "pulmonary nodule", "indocyanine green (or ICG)" and "pulmonary segment", "indocyanine green (or ICG)" and "gastroesophageal anastomosis", "indocyanine green (or ICG)" and "pulmonary bulla", "indocyanine green (or ICG)" and "thoracic duct", "indocyanine green (or ICG)" and "sentinel lymph node". We almost select the latest 5-year literatures about ICG NIRF technology used in thoracic surgery to guarantee the advancement of our review paper.

\section{Pulmonary nodule localization}

For sub-cm pulmonary nodules, precise positioning during VATS still presents significant challenges. Conventional hook-wire, spring coil, and methylene blue injection have complications such as hook-wire slip, dye dispersion, pneumothorax, and air embolism. Studies have manifested that because of the enhanced permeability and retention (EPR) effect (ICG is retained in the tumor tissue due to increased vascular permeability), ICG can concentrate in tumor tissue (1), but does not damage lung tissue and affect pathological diagnosis. Anayama et al. injected ICG $(0.3 \mathrm{~mL}$, $0.15 \mathrm{mg} / \mathrm{mL}$ ) around the pulmonary nodules of pigs through magnetic navigation bronchoscopy. After 6 hours, the isolated fluorescent region was successfully observed under fluorescent thoracoscopy, and pathological anatomy confirmed as isolated pulmonary nodule (2). Clinical practice has proven that percutaneous computed tomography (CT)guided pulmonary nodules positioning by ICG is safe and feasible without serious complications (3). Wen et al. studied the median time required for NIRF thoracoscopy localization was $15 \mathrm{~min}$ [interquartile range (IQR), 13-16 min; ICG quantity: $0.3-0.5 \mathrm{~mL}$; dye concentration: $0.125 \mathrm{mg} / \mathrm{mL}$ ]; it is recommended that surgery be performed within 3 hours after labeling to prevent ICG from spreading into the pulmonary parenchyma and affect the visualization of pulmonary nodules (4). Anayama et al. thought that the transbronchial approach can provide a similar success rate compared to percutaneous positioning (ICG: $2.5 \mathrm{mg} / \mathrm{mL}, 10 \mathrm{~mL}$ ). Transbronchial localization does not injure the visceral pleura, reducing 
the incidence of pneumothorax. In addition, bronchoscopy can locate sites that are difficult to reach by percutaneous puncture, such as the mediastinal side and the part that is blocked by the scapula $(2,5)$. Moreover, ICG can also be administered intravenously. Okusanya et al. injected ICG $(5 \mathrm{mg} / \mathrm{kg}$ ) intravenously $24 \mathrm{~h}$ before surgery, the nodule detection rate was $88.9 \%$ [16/18], the NIRF imaging also identified five additional sub-cm nodules, three metastatic adenocarcinomas and two metastatic sarcomas (6). Mao et al. showed that the mean near-infrared signal to background ratio of the pulmonary nodules by intravenous ICG $(5 \mathrm{mg} / \mathrm{kg})$ was $3.29 \pm 1.81$, the sensitivity and positive predictive value of ICG NIRF imaging were $88.7 \%$ and $92.6 \%$, respectively (7). The sensitivity of this method is not affected by factors such as nodules size, metabolic activity, histological type, etc., and small nodules that are neglected by CT can be also visualized; however, this technique has limitations on the depth of nodules, which is reported in the literature as $1.3-2.0 \mathrm{~cm}(4,6)$. It is unable to detect ICG fluorescence because of incomplete deflation of the lung caused by inadequate double-lumen bronchial tube placement. In lungs with air volume retention, it is difficult to see ICG fluorescence (2). Therefore, it is particularly important to develop new imaging techniques to discover deep nodules and to probe new contrast agents to classify different histological nodules (8). According to the economics, it is costly to install the guided therapeutic operating room with a CT scanner and cone-beam CT for intraoperative fluoroscopy, and these technologies may not be readily available in the operating room for thoracic surgeons.

\section{Precise demarcation of segmental plane}

Optimal identification of the intersegmental plane can be challenging during thoracoscopic anatomical segmentectomy for lung cancer. Previously, the inflation test or the post-inflation collapse test had disadvantages because there was partial ventilation between the alveolar of different pulmonary segments, and the boundaries of the lung segments could not be accurately displayed. Moreover, the inflation and post-inflation collapse test affected the intrathoracic space and impeded surgical procedures, especially in patients with emphysema. Animal studies have shown that the pulmonary segmental plane can be clearly displayed under fluorescent thoracoscopy after intravascular injection of ICG (9). In recent years, ICG NIRF imaging technology has also been applied clinically. According to
Mun's study, ICG-fluorescence (ICGF) by intravenous systemic injection $(0.25 \mathrm{mg} / \mathrm{kg})$ provided demarcation of sufficient clarity and duration to mark the lung surface in 19 patients (95\%). The segmental plane appeared after $20 \mathrm{~s}(10-100 \mathrm{~s})$ of ICG injection, and ICGF lasted $180 \mathrm{~s}$ (90-300 s). Contrast index (CI) peaked $30 \mathrm{~s}$ after the appearance of ICGF and decreased over time. Effective contrast lasted $70 \mathrm{~s}(30-116 \mathrm{~s})$, which was sufficient to mark the line of demarcation (10). Kasai et al. compared the identifying efficacy of pulmonary intersegmental plane with the use of ICG between 2-wavelength (940 and $805 \mathrm{~nm}$ ) and 1-wavelength (780 and $830 \mathrm{~nm}$ ) infrared thoracoscopy, they found the 1-wavelength method can provide longer staining duration than that of the 2-wavelength method ( 0.5 vs. $3.0 \mathrm{mg} / \mathrm{kg}, 370$ vs. $220 \mathrm{~s}, \mathrm{P}=0.0001$ ) (11). Some scholars have pointed out that transbronchial injection of ICG may be superior to intravenous injection. Because the ICG is rapidly metabolized by the liver through the bronchial venous circulation after intravenous injection, and the segmental plane imaging duration is shortened significantly. Sekine $\mathrm{et} \mathrm{al}$. injected ICG (20 to $30 \mathrm{~mL}$ of 5 -fold salinediluted ICG) into the target pulmonary segment through the trachea, and visualized the segmental plane through fluorescent thoracoscopy to accurately dissect the target segment (12). However, Elkhouly et al. proposed that this method has certain defects (13). First, the ICG may return from the target bronchus to the bronchial tree, interfering with the identification of the segmental plane; second, injecting the ICG into bronchus of the target segment may cause the tumor cells to spread across the target segment to other segments. Therefore, they firstly disconnected the blood vessels and bronchus of the target segment, and then injected ICG (30 mL of $10 \%$ saline-diluted ICG) through the trachea, and displayed segmental plane by means of fluorescent thoracoscopy within 3-5 minutes, thus avoiding the deficiency of the previous method. There are some limitations of ICGF in identification of the intersegmental plane. A potential issue is the spread of ICG to adjacent regions during operation. Another is the amount of ICG given and the IRT system are not fixed, as it is important to empirically search for the optimal dose and system parameters.

\section{Evaluation of blood supply of gastroesophageal anastomosis}

Anastomotic leakage is considered to be an independent risk factor of postoperative mortality in esophageal cancer, 
and blood supply during gastroesophageal remodeling is an important factor affecting anastomotic leakage. In recent years, more and more studies report that ICG fluorescence technology can provide real-time visualization of blood supply and guide the selection of anastomotic sites in the gastroesophageal anastomosis. Koyanagi et al. demonstrated that intraoperative evaluation of blood flow speed through ICG fluorescence technology in the gastric conduit wall is an effective means of predicting the risk of anastomotic leakage after esophagectomy (14). Rino et al. found the blood supply route was mostly located in the greater omentum beside the splenic hilum on ICG $(2.5 \mathrm{mg})$ fluorescence imaging (15). Kumagai et al. proposed that it is safe to perform anastomosis in the area enhanced by ICG $(2.5 \mathrm{mg})$ fluorescein angiography within $60 \mathrm{~s}$, resected areas with a delayed enhancement time of more than $90 \mathrm{~s}$ can avoid gastric conduit necrosis. The 90 -s rule is a safe and effective method for deciding the site of anastomosis (16). Meta-analysis also demonstrated that microvascular perfusion is associated with anastomotic leakage, application of ICG NIRF technology as an intraoperative tool to visualize microvascular perfusion and select gastroesophageal anastomotic sites to reduce the incidence of anastomotic leakage and improve the safety of surgery is feasible $(17,18)$. One limitation is that blood flow in the gastric tube is influenced by the patient's general condition during the perioperative period. Another is the assessment of fluorescence intensity remains somehow subjective with this technology. Future imaging and software enhancements are warranted to achieve an objective method of fluorescence quantification.

\section{Tracing SLNs}

SLNs dissection has become an integral part of the scale of LNs dissection for solid malignancies (such as breast cancer, malignant melanoma, etc.). For the evaluation of SLNs in lung cancer, the current clinical application is limited, mainly due to the presence of carbon deposition in the intrathoracic lymph nodes. There is no obvious contrast after conventional dyeing, resulting in poor recognition of SLNs in lung cancer. Gilmore et al. assessed the safety and feasibility of near-infrared imaging using ICG $(3.8 \mu \mathrm{g})$ for SLN identification in non-small cell lung cancer (NSCLC) in 29 patients. After 5 to 20 minutes, the lymph nodes showed successfully (19). In subsequent clinical trials, they enrolled 46 patients with considered or confirmed lung cancer, and 38 underwent intraoperative application of ICG for localization of SLNs. The results showed that the SLNs identification was unclear when the ICG dose was less than $600 \mu \mathrm{g}$; the lymphatic migration and lymph node detection rates were increased at doses of 600,800 , 1,000 , and $2,500 \mu \mathrm{g}$, which were $33 \%, 40 \%, 80 \%$, and $100 \%$, respectively, and there were no adverse reactions (20). Yamashita et al. detected SLNs about 10 minutes after injection of $2 \mathrm{~mL}(5 \mathrm{mg} / \mathrm{mL})$ of ICG around the tumor, with a total recognition rate of $80.3 \%$ [49/61] and a false negative rate of $2.0 \%$ [1/49], the overall accuracy rate was $78.7 \%$ [48/61]. The causes of failure were technical failure such as ICG leakage from injection sites, intrathoracic adhesion, incomplete fissure of lobes, and emphysematous lung (21). Oh et al. demonstrated ICG-neomannosyl human serum albumin (ICG:HSA) showed a significantly improved signal-to-background ratio (SBR) compared with ICG alone, ICG:HSA accumulated in the SLNs by uptake and retention through the mannose-specific receptors on macrophages. Intraoperative color and fluorescence imaging system (ICFIS) successfully assisted SLNs mapping despite low near-infrared light transmission in the commercial thoracoscope. They anticipated that ICG:HSA and thoracoscopic ICFIS can be translated to clinical trials in the near future (22).

\section{Thoracic duct (TD) imaging}

Chylothorax is a common complication of thoracic surgery, especially in esophagectomy. Trauma of the TD often leads to unplanned secondary surgery for TD ligation. Preoperative oral or nasal feeding of whole milk, CT lymphography (CTL) or methylene blue injection can help trace the leakage of the chyle, but there are shortcomings such as poor contrast, bad reproducibility, and coverage of mediastinal fat. Animal models have demonstrated that NIRF technique provide sensitive, continuous, realtime TD anatomy and functional imaging in thoracotomy and VATS, and are superior to CTL and methylene blue injection $(23,24)$. Using a similar method, Yang et al. injected ICG $(0.2 \mathrm{mg} / \mathrm{kg})$ subcutaneously into the bilateral inguinal region approximately $30 \mathrm{~min}$ before surgery, and with the guidance of real-time fluorescence lymphography, the fistulas and the main trunk of the TD were identified and double ligated (25). Kaburagi et al. and Matsutani et al. reported two patients [one patient was injected $2 \mathrm{~mL}$ of ICG solution $(0.5 \%)$ into the mesentery of the small bowel, another was injected subcutaneously of $1.5-\mathrm{mL}$ ICG solution $(0.5 \%)$ into the inguinal region] were performed 
by abdominal lymphatic ligation with the aid of ICG fluorescence navigation and got ahead (26,27). Tan et al. successfully traced the TD by injecting ICG intradermally into a 5-week-old child to help treat chylothorax after Norwood surgery (28). Shirotsuki et al. reported ICGNIRF imaging was performed in 10 newborns (11 surgeries) with first tracheoesophageal fistula (TEF) operation or with persistent postoperative chylothorax after TEF operation. They performed NIRF imaging 1 hour after injection of ICG $(0.025 \mathrm{mg})$ between the newborn's toes, and all the children successfully found the fistula and sutured with no related complications (29). Using ICG-NIRF imaging during operation allows the visualization of the TD, which may allow preservation of the duct and easier ligation for duct injuries.

\section{Complete resection of pulmonary bulla}

Pulmonary bulla resection under VATS is considered the preferred treatment for most patients with spontaneous pneumothorax. Compared with traditional thoracotomy, bullectomy under VATS has the advantages of less bleeding, milder postoperative pain, and shorter postoperative hospitalization; however, studies have shown that the recurrence rate of minimally invasive surgery is higher, mainly due to bulla residual, especially in patients who are difficult to distinguish the edge of bulla. Compared with adjacent normal lung tissue, bulla or emphysema lesions have lower tissue density and less blood flow, the concentration distribution of ICG after intravenous injection is lower. A sudden drop in ICG concentration can be visualized in real time by NIRF imaging to clearly distinguish between bulla and normal lung tissue. Gotoh et al. reported that the emphysema area can be detected by fluorescent thoracoscopic surgery after intravenous injection of ICG in animal model (30). Subsequently, they applied this technique (ICG: $3.0 \mathrm{mg} / \mathrm{kg}$ ) to the clinic and succeeded (31). Matsumoto et al. used ICG $(0.5 \mathrm{mg} / \mathrm{kg})$ to precisely delineate the diseased lesion using NIRF imaging in a 75-year-old woman with a large emphysematous lesion in the right lower lobe (32). $\mathrm{Li}$ et al. detected a fluorescent signal in normal lung tissue $10.5 \mathrm{~s}$ after ICG bolus (average 10-11 s) and lasted for up to $525 \mathrm{~s}$ (mean, 480-570 s). The bullous lesions showed an obviously decreased fluorescent densities comparing to adjacent normal tissue. The maximum SBR of 0.2 and $0.6 \mathrm{mg} / \mathrm{kg}$ ICG groups between normal lung and bullous lesions were 5.77 and 6.32 , respectively (33). None of the above studies have complications associated with surgery, demonstrating that ICG fluorescence thoracoscopic techniques have higher safety and better application prospects in the treatment of pulmonary bulla. However, some tissues, like the fibrous tissue, which have decreased blood flow may present as low fluorescent signal as bullous tissues, so it would need more studies to apply this technology into real clinical practice. In addition, more ICG doses should be tested before the optimal dose was defined.

\section{Recognition of neural tissue}

In recent years, ICG-NIRF imaging technique has also been applied in nerve tissue labeling and protection during thoracic surgery. Wagner et al. reported that fluorescence imaging in unilateral right robotic thymectomy $(6.25 \mathrm{mg}$ of ICG followed by a $10 \mathrm{~mL}$ bolus of normal saline) can help identify the contralateral phrenic nerve by fluorescing the pericardiophrenic vessels (34). This technology has the potential to maximize thymic tissue resection with a unilateral approach while reducing operative time and nerve injury. Weng et al. discovered that the thoracic sympathetic ganglions, including the stellate ganglion (SG) under ICG fluorescence thoracoscopic surgery (35). This new finding may be beneficial for the accurate resection of sympathetic nerves in palmar hyperhidrosis. He et al. also confirmed from animal experiments (10 $\mathrm{mg} / \mathrm{kg}$ of ICG) to human practice $(5 \mathrm{mg} / \mathrm{kg}$ of ICG) that ICG fluorescent thoracoscopy can locate the ganglia from the SG to the sixth thoracic ganglion (T6), as well as the chains between these ganglia in all patients with a high SBR value of 3.26 (standard deviation: 0.57) (36). The technique may replace the rib-oriented method as standard practice for mapping the thoracic sympathetic nerves. However, the nerve imaging mechanism is unclear and remains to be confirmed by further animal experiments.

\section{Application in other fields}

ICG NIRF imaging technology can also be applied in others fields, such as tracheobronchial surgery (37). Schweiger et al. injected ICG intravenously at $0.2 \mathrm{mg} / \mathrm{kg}$ body weight to assess the anastomotic suture line after the operation. They think, in the future, it might contribute to an early detection of anastomotic failure and reduce morbidity and mortality after airway surgery (38). Kennedy et al. suggest that ICG is effective in identifying mesothelioma in humans and a murine model (39). In mapping for surgical resection 
of lung metastasis, ICG NIRF imaging technology is also useful $(40,41)$.

\section{Conclusions}

With the prolongation of clinical application time of ICG real-time fluorescein imaging technology, more and more new means have been developed. A large number of animal experiments and clinical practice demonstrate the details and effects of real-time fluorescence imaging technology applied in thoracic surgery, which provides an evidencebased basis for solving the problems mentioned in this paper. It is believed that the ICG NIRF imaging technology in thoracic surgery will become more and more extensive. In the future, more ICG doses should be tested to define the optimal dose. Moreover, future imaging and software enhancements are warranted to achieve an objective method of fluorescence quantification.

\section{Acknowledgments}

Funding: None.

\section{Footnote}

Reporting Checklist: The authors have completed the Narrative Review reporting checklist. Available at https:// ccts.amegroups.com/article/view/10.21037/ccts-19-84/rc

Peer Review File: Available at https://ccts.amegroups.com/ article/view/10.21037/ccts-19-84/prf

Conflicts of Interest: All authors have completed the ICMJE uniform disclosure form (available at https://ccts. amegroups.com/article/view/10.21037/ccts-19-84/coif). The authors have no conflicts of interest to declare.

Ethical Statement: The authors are accountable for all aspects of the work in ensuring that questions related to the accuracy or integrity of any part of the work are appropriately investigated and resolved.

Open Access Statement: This is an Open Access article distributed in accordance with the Creative Commons Attribution-NonCommercial-NoDerivs 4.0 International License (CC BY-NC-ND 4.0), which permits the noncommercial replication and distribution of the article with the strict proviso that no changes or edits are made and the original work is properly cited (including links to both the formal publication through the relevant DOI and the license). See: https://creativecommons.org/licenses/by-nc-nd/4.0/.

\section{References}

1. Ishizawa T, Masuda K, Urano Y, et al. Mechanistic background and clinical applications of indocyanine green fluorescence imaging of hepatocellular carcinoma. Ann Surg Oncol 2014;21:440-8.

2. Anayama T, Qiu J, Chan H, et al. Localization of pulmonary nodules using navigation bronchoscope and a near-infrared fluorescence thoracoscope. Ann Thorac Surg 2015;99:224-30.

3. Nagai K, Kuriyama K, Inoue A, et al. Computed tomography-guided preoperative localization of small lung nodules with indocyanine green. Acta Radiol 2018;59:830-5.

4. Wen CT, Liu YY, Fang HY, et al. Image-guided videoassisted thoracoscopic small lung tumor resection using near-infrared marking. Surg Endosc 2018;32:4673-80.

5. Anayama T, Hirohashi K, Miyazaki R, et al. Near-infrared dye marking for thoracoscopic resection of small-sized pulmonary nodules: comparison of percutaneous and bronchoscopic injection techniques. J Cardiothorac Surg 2018;13:5.

6. Okusanya OT, Holt D, Heitjan D, et al. Intraoperative near-infrared imaging can identify pulmonary nodules. Ann Thorac Surg 2014;98:1223-30.

7. Mao Y, Chi C, Yang F, et al. The identification of subcentimetre nodules by near-infrared fluorescence thoracoscopic systems in pulmonary resection surgeries. Eur J Cardiothorac Surg 2017;52:1190-6.

8. Newton AD, Kennedy GT, Predina JD, et al. Intraoperative molecular imaging to identify lung adenocarcinomas. J Thorac Dis 2016;8:S697-704.

9. Oh Y, Quan YH, Choi Y, et al. Intraoperative combined color and fluorescent images-based sentinel node mapping in the porcine lung: comparison of indocyanine green with or without albumin premixing. J Thorac Cardiovasc Surg 2013;146:1509-15.

10. Mun M, Okumura S, Nakao M, et al. Indocyanine green fluorescence-navigated thoracoscopic anatomical segmentectomy. J Vis Surg 2017;3:80.

11. Kasai Y, Tarumi S, Chang SS, et al. Clinical trial of new methods for identifying lung intersegmental borders using infrared thoracoscopy with indocyanine green: comparative analysis of 2- and 1-wavelength methods. Eur 
J Cardiothorac Surg 2013;44:1103-7.

12. Sekine $\mathrm{Y}, \mathrm{Ko} \mathrm{E}$, Oishi $\mathrm{H}$, et al. A simple and effective technique for identification of intersegmental planes by infrared thoracoscopy after transbronchial injection of indocyanine green. J Thorac Cardiovasc Surg 2012;143:1330-5.

13. Elkhouly AG, Cristino B, Pompeo E. A new method of infrared-fluorescence-enhanced thoracoscopic segmentectomy. Asian Cardiovasc Thorac Ann 2018;26:247-9.

14. Koyanagi K, Ozawa S, Oguma J, et al. Blood flow speed of the gastric conduit assessed by indocyanine green fluorescence: New predictive evaluation of anastomotic leakage after esophagectomy. Medicine (Baltimore) 2016;95:e4386.

15. Rino Y, Yukawa N, Sato T, et al. Visualization of blood supply route to the reconstructed stomach by indocyanine green fluorescence imaging during esophagectomy. BMC Med Imaging 2014;14:18.

16. Kumagai Y, Hatano S, Sobajima J, et al. Indocyanine green fluorescence angiography of the reconstructed gastric tube during esophagectomy: efficacy of the 90 -second rule. Dis Esophagus 2018;31. doi: 10.1093/dote/doy052.

17. Ladak F, Dang JT, Switzer N, et al. Indocyanine green for the prevention of anastomotic leaks following esophagectomy: a meta-analysis. Surg Endosc 2019;33:384-94.

18. Slooter MD, Eshuis WJ, Cuesta MA, et al. Fluorescent imaging using indocyanine green during esophagectomy to prevent surgical morbidity: a systematic review and metaanalysis. J Thorac Dis 2019;11:S755-65.

19. Gilmore DM, Khullar OV, Colson YL. Developing intrathoracic sentinel lymph node mapping with nearinfrared fluorescent imaging in non-small cell lung cancer. J Thorac Cardiovasc Surg 2012;144:S80-4.

20. Gilmore DM, Khullar OV, Jaklitsch MT, et al. Identification of metastatic nodal disease in a phase 1 dose-escalation trial of intraoperative sentinel lymph node mapping in non-small cell lung cancer using near-infrared imaging. J Thorac Cardiovasc Surg 2013;146:562-70.

21. Yamashita S, Tokuishi K, Miyawaki M, et al. Sentinel node navigation surgery by thoracoscopic fluorescence imaging system and molecular examination in non-small cell lung cancer. Ann Surg Oncol 2012;19:728-33.

22. Oh Y, Lee YS, Quan YH, et al. Thoracoscopic color and fluorescence imaging system for sentinel lymph node mapping in porcine lung using indocyanine greenneomannosyl human serum albumin: intraoperative image-guided sentinel nodes navigation. Ann Surg Oncol 2014;21:1182-8.

23. Ashitate Y, Tanaka E, Stockdale A, et al. Near-infrared fluorescence imaging of thoracic duct anatomy and function in open surgery and video-assisted thoracic surgery. J Thorac Cardiovasc Surg 2011;142:31-8.e1-2.

24. Steffey MA, Mayhew PD. Use of direct near-infrared fluorescent lymphography for thoracoscopic thoracic duct identification in 15 dogs with chylothorax. Vet Surg 2018;47:267-76.

25. Yang F, Zhou J, Li H, et al. Near-infrared fluorescenceguided thoracoscopic surgical intervention for postoperative chylothorax. Interact Cardiovasc Thorac Surg 2018;26:171-5.

26. Kaburagi T, Takeuchi H, Oyama T, et al. Intraoperative fluorescence lymphography using indocyanine green in a patient with chylothorax after esophagectomy: report of a case. Surg Today 2013;43:206-10.

27. Matsutani T, Hirakata A, Nomura T, et al. Transabdominal approach for chylorrhea after esophagectomy by using fluorescence navigation with indocyanine green. Case Rep Surg 2014;2014:464017.

28. Tan IC, Balaguru D, Rasmussen JC, et al. Investigational lymphatic imaging at the bedside in a pediatric postoperative chylothorax patient. Pediatr Cardiol 2014;35:1295-300.

29. Shirotsuki R, Uchida H, Tanaka Y, et al. Novel thoracoscopic navigation surgery for neonatal chylothorax using indocyanine-green fluorescent lymphography. J Pediatr Surg 2018;53:1246-9.

30. Gotoh M, Okamoto T, Yamamoto Y, et al. Development of a canine model of pulmonary emphysema and imaging of the emphysematous lung with infrared thoracoscopy. J Thorac Cardiovasc Surg 2003;126:1916-21.

31. Gotoh M, Yamamoto Y, Igai H, et al. Clinical application of infrared thoracoscopy to detect bullous or emphysematous lesions of the lung. J Thorac Cardiovasc Surg 2007;134:1498-501.

32. Matsumoto K, Sano I, Taniguchi H, et al. Thoracoscopic surgery for lung emphysema using an infrared camera. J Cardiothorac Surg 2013;8:134.

33. Li H, Zhou J, Chi C, et al. Clinical application of near-infrared thoracoscope with indocyanine green in video-assisted thoracoscopic bullectomy. J Thorac Dis 2016;8:1841-5.

34. Wagner OJ, Louie BE, Vallieres E, et al. Near-infrared fluorescence imaging can help identify the contralateral phrenic nerve during robotic thymectomy. Ann Thorac 
Surg 2012;94:622-5.

35. Weng W, Liu Y, Zhou J, et al. Thoracoscopic Indocyanine Green Near-Infrared Fluorescence for Thoracic Sympathetic Ganglions. Ann Thorac Surg 2016;101:2394.

36. He K, Zhou J, Yang F, et al. Near-infrared Intraoperative Imaging of Thoracic Sympathetic Nerves: From Preclinical Study to Clinical Trial. Theranostics 2018;8:304-13.

37. Akopov AL, Papayan GV, Efimov AN, et al. Infrared Fluorescent Angiography during Experimental Trachea Transplantation. Bull Exp Biol Med 2018;164:519-22.

38. Schweiger T, Schwarz S, Traxler D et al. Bronchoscopic Indocyanine Green Fluorescence Imaging of the Anastomotic Perfusion After Tracheal Surgery. Ann

doi: $10.21037 /$ ccts-19-84

Cite this article as: Wang F, Weng Y, Geng J, Zhu J. A narrative review of indocyanine green near-infrared fluorescence imaging technique: a new application in thoracic surgery. Curr Chall Thorac Surg 2021;3:35.
Thorac Surg 2016;101:1943-9.

39. Kennedy GT, Newton A, Predina J et al. Intraoperative near-infrared imaging of mesothelioma. Transl Lung Cancer Res 2017;6:279-84.

40. Predina JD, NewtonAD, Corbett C et al. Near-infrared intraoperative imaging for minimally invasive pulmonary metastasectomy for sarcomas. J Thorac Cardiovasc Surg 2019;157:2061-9.

41. Miyazaki $Y$, Kurata $M$, Oshiro $Y$ et al. Indocyanine green fluorescence-navigated laparoscopic metastasectomy for peritoneal metastasis of hepatocellular carcinoma: a case report. Surg Case Rep 2018;4:130. 